\title{
Leave no one behind: ensuring access to COVID-19 vaccines for refugee and displaced populations
}

\author{
Ensuring access to vaccines against COVID-19 for refugee and displaced populations and addressing health \\ inequities are vital for an effective pandemic response.
}

\author{
Monette Zard, Ling San Lau, Diana M. Bowser, Fouad M. Fouad, Diego I. Lucumí, Goleen Samari, \\ Arturo Harker, Donald S. Shepard, Wu Zeng, Rachel T. Moresky, Mhd Nour Audi, Claire M. Greene \\ and S. Patrick Kachur
}

$\mathrm{T}$ he rising toll of the COVID-19 pandemic and concerns about emerging SARS-CoV-2 variants have underscored the urgency of a rapid, equitable and inclusive rollout of vaccines against COVID-19. However, as the world grapples with supply challenges and inequitable vaccine access on local and global scales, marginalized groups, particularly refugees, internally displaced persons and stateless persons, will face a double burden of access, even within countries that are themselves marginalized on the global stage. Worldwide, over $80 \%$ of refugees and nearly all internally displaced persons are hosted by low- and middle-income countries ${ }^{1}-$ nations that are at the end of the line for doses of vaccines against COVID-19, and where vaccine distribution will expose the fissures in overburdened and weak health systems. Refugees and other displaced populations in these settings face additional barriers to vaccine access-in both policy and practice-that must be acknowledged and overcome. In fragile settings with weak governance, competition for scarce vaccines against COVID-19 may heighten tensions and exacerbate conflict, while unequal access raises the prospect that populations will move in an effort to access vaccines that are not available in their country or region. A coordinated, comprehensive and global approach to vaccine access is vital, in contrast to the highly politicized and nationalist discourse that has characterized the pandemic response thus far. Science and need, not wealth, nationality or legal status, should guide vaccine allocation and prioritization.

\section{Ensuring equitable access in a context of vaccine scarcity}

Despite calls for global cooperation and the declaration by United Nations International
Children's Emergency Fund Executive Director Henrietta Fore and World Health Organization Director-General Tedros Adhanom Ghebreyesus that "in the COVID19 vaccine race, we either win together or lose together,"2 the allocation and distribution of vaccines has been neither equitable nor inclusive. As of $30 \mathrm{March}$ 2021, high- and upper-middle-income countries received $86 \%$ of the vaccine doses delivered worldwide, whereas only $0.1 \%$ of doses have been delivered in low-income countries $^{2}$. In the race to secure scarce vaccine doses, wealthier countries have leveraged advance purchase agreements to outcompete countries with fewer resources ${ }^{3}$. The COVAX global vaccine facility, an initiative jointly led by the World Health Organization, the Coalition for Epidemic Preparedness Innovations and Gavi, the Vaccine Alliance, aims to broaden the coverage of vaccines against SARS-CoV-2 in low- and middle-income countries, and includes a small 'humanitarian buffer' (around 5\% of the total available vaccine doses) for humanitarian use, including the vaccination of refugees ${ }^{4}$. However, this is wholly insufficient: the total two billion vaccine doses targeted by the end of 2021 will cover only $20 \%$ of the participating countries' populations, at most ${ }^{5}$. Current projections indicate that poorer countries may not be able to widely vaccinate their populations until 2023 (ref. ${ }^{6}$ ).

Against this backdrop of global inequality, refugees and displaced populations confront additional barriers to vaccine access and healthcare services more generally, within the countries in which they reside. Thus far, 110 countries have explicitly included refugees in their vaccine-distribution plans ${ }^{7}$. However, issues of cost (both direct healthcare costs and additional out-of-pocket costs associated with seeking care), discrimination and stigma, as well as distrust of authorities and fears related to disclosure of immigration status, may deter displaced persons from seeking vaccination, even when they are eligible ${ }^{8}$. In many countries, a lack of reliable data on displaced populations will make it difficult to identify people who are eligible for vaccination, while mobility and repeated displacement present challenges to continuity of care, including follow-up for subsequent vaccine doses ${ }^{9}$, where required. Timely, reliable information about vaccines-including information on their efficacy and safety, and how to access them-is essential for promoting vaccine acceptance and trust. However, in many displacement settings, such as the Rohingya refugee settlements in Cox's Bazar, in Bangladesh, access to information and telecommunications is often restricted.

Displaced populations are often served by weak, fragmented and donor-dependent health systems that grapple with a multitude of health needs, many of which have been complicated or exacerbated by the COVID-19 pandemic . $^{8}$ These include outbreaks of communicable disease, such as the simultaneous outbreaks of measles and Ebola besetting the Democratic Republic of the Congo and Guinea, malaria, malnutrition, routine childhood immunization programs, sexual and reproductive health, mental health, and chronic conditions such as HIV infection and tuberculosis ${ }^{8,10-12}$. Weak health infrastructures, including limited cold-chain capabilities, shortages of trained healthcare workers ${ }^{10}$ and fragmented health-information systems $\mathrm{s}^{10}$, pose perennial problems, particularly in remote areas where refugee camps are often located, and these challenges will complicate any future vaccine rollout. In conflict 
settings, violence, insecurity and attacks against health facilities and health personnel are key challenges ${ }^{13}$.

Recognizing that a narrow focus on emergency vaccine programs may destabilize fragile health systems and disrupt other essential health services, policymakers and donors need to seize the opportunity of this pandemic to strengthen health systems more broadly and sustainably, to better respond to the challenges of COVID-19 while addressing the comprehensive health needs of refugees and host populations. A study from six low- and middle-income countries showed that the introduction of new vaccines improved staff training and disease surveillance, and increased domestic and external funding. In addition, introducing new vaccines strengthened collaborations among the different national agencies ${ }^{14}$.

\section{Legal status should not determine vaccine access}

Amid intense nationalism, governments may yield to pressure to prioritize vaccine access for their own citizens, even when this runs contrary to public-health goals and may prolong the pandemic. Widespread tensions ${ }^{15}$ over vaccine prioritization are magnified in fragile settings in which the introduction of vaccines against COVID-19 will add to an already volatile mix of weak governance, corruption and intra-community strife, and they may stoke concerns about incentivizing further cross-border movements. In Lebanon, host to more than 1.7 million Syrian and Palestinian refugees, the government has committed in principle to equitable vaccine access for all, yet members of the president's own party, disturbed by the prospect of refugees accessing vaccines, have propagated xenophobic hashtags ${ }^{16}$. In Syria, where the pandemic has taken a considerable toll, advocacy organizations have expressed concerns that the government may continue its pattern of rewarding allies and punishing enemies when distributing vaccine doses ${ }^{17}$. These trends are likely to play out across conflict zones around the world, with the potential to spark new sources of conflict ${ }^{18}$. In late 2020, President Ivan Duque of Colombia, which is currently hosting 1.7 million Venezuelan migrants (the majority of whom are undocumented), sparked controversy when he justified largely excluding this population from the national vaccination program to avoid a "stampede" across the border to access Colombia's vaccines against COVID-19 ${ }^{19}$. In a positive step forward, Colombia announced a regularization program in February 2021 that will grant temporary protected status to nearly one million Venezuelans, entitling them to access the national healthcare system and, by extension, vaccines against COVID-19 ${ }^{20}$. That example notwithstanding, legal status should have no place in decisions about vaccine access, and relying on regularization as a route to vaccination will unacceptably delay the protective effects for migrants and refugees, particularly in groups at higher risk.

Although the complexity and scale of the rollout of vaccines against COVID-19 is formidable, policymakers and public-health officials can draw lessons from experience managing conditions such as HIV infection and tuberculosis among mobile populations, as well as previous large-scale vaccination campaigns in humanitarian settings. The global polio-eradication program has brought the world to the brink of eradicating polio, through intensive efforts to vaccinate mobile, conflict-affected and other marginalized populations. Valuable lessons for increasing vaccine coverage among these populations include strengthening vaccination and surveillance activities at border crossings, transit hubs and other access points, such as refugee camps, settlements for internally displaced persons, markets and religious gatherings; integrating vaccination activities with essential humanitarian activities, including food and medicine distribution and nutritional assessments; using geographic-information-systems technology to identify potentially eligible populations in remote areas, inform vaccine program planning, and track migration patterns; building flexibility into vaccine scheduling and dosing; and negotiating with parties to the conflict to ensure secure access to vaccine-eligible populations ${ }^{21}$. For example, El Salvador's "days of tranquility" allowed vaccination against polio to proceed despite civil unrest and were credited with building a "bridge for peace."22

Engaging and listening to communities is also critical, through risk communication and education, mobilizing trusted community and religious leaders, and involving community members, including displaced populations, in vaccine activities $^{21,23}$. A MEASURE Evaluation study emphasized the importance of a cross-border health-systems approach to improving care for those with HIV and tuberculosis among mobile populations in East Africa, recommending regional and cross-facility linkage of patient records, and measures to reduce barriers to health-service access among mobile populations ${ }^{24}$. Investments to implement and strengthen mobile health data-collection tools, such as the regional vaccination card developed for displaced Venezuelans in Latin America, and the District Health Information System II software used by national health systems in many lower-income countries, have been proposed as potential methods for documenting and tracking immunization through displacement ${ }^{9}$. Models of care for people living with HIV have also demonstrated the value of innovative strategies such as mobile outreach and community-based service-delivery and drug-distribution points, including key transit hubs and migration destinations ${ }^{25}$.

\section{A call for stronger, fairer health systems}

Ensuring that those populations most marginalized among us are able to access vaccines against COVID-19 is not only a moral imperative but also vital for our collective health and well-being. As a global community, we have the ability to increase vaccine reach among displaced populations and to leverage investments in the rollout of vaccines against COVID-19 to strengthen health infrastructure for all, while meaningfully including displaced communities in the process. As the world confronts one of the most formidable public-health challenges in recent history, how we respond today will determine not only the course of this pandemic but also who benefits from public-health advances for years to come. We may further entrench the inequities and distrust experienced by refugees and displaced populations around the world, or, by recognizing and confronting these inequities, we can build stronger, fairer health systems that are better prepared to respond to COVID-19 and future health emergencies.

Editorial note: The Spanish translation of this Comment, provided as Supplementary Information, has not been edited by the publisher.

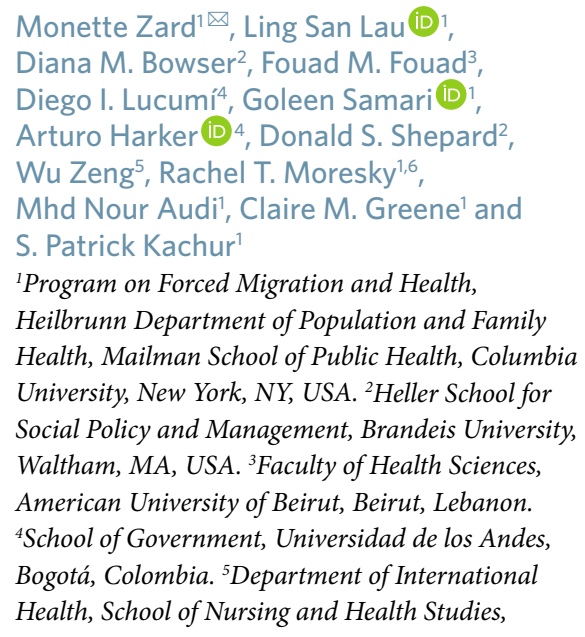


Georgetown University, Washington, DC, USA.

${ }^{6}$ Department of Emergency Medicine, Columbia University Irving Medical Center, New York, NY, USA.

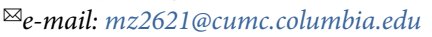

Published online: 19 April 2021

https://doi.org/10.1038/s41591-021-01328-3

References

1. UNHCR. https://www.unhcr.org/refugee-statistics/ (accessed 23 February 2021)

2. Collins, K. \& Holder, J. https://www.nytimes.com/interactive/ 2021/03/31/world/global-vaccine-supply-inequity.html The New York Times (31 March 2021).

3. Phelan, A. L., Eccleston-Turner, M., Rourke, M., Maleche, A. \& Wang, C. Lancet 396, 800-802 (2020)

4. Berkley, S. Gavi, the Vaccine Alliance https://www.gavi.org/ vaccineswork/covax-explained (2020).

5. Figueroa, J. P. et al. Lancet 397, 562-564 (2021).

6. The Economist Intelligence Unit. The Economist https://www.eiu. com/n/85-poor-countries-will-not-have-access-to-coronavirusvaccines/ (27 January 2021)

7. The New Humanitarian https://www.thenewhumanitarian.org/ maps-and-graphics/2021/coronavirus-humanitarian-updatemap-data-vaccine-aid-response (2021).

8. The International Federation of Red Cross and Red Crescent Societies. https://media.ifrc.org/ifrc/document/least-protectedaffected-migrants-refugees-facing-extraordinary-risks-covid-19pandemic/ (10 September 2020).

9. Nair, S. Refugees International https://www.refugeesinternational. org/reports/2020/12/29/vaccine-authorization-is-just-thebeginning-of-the-journey-to-protecting-displaced-populationsfrom-covid-19 (29 December 2020).
10. Rohan, H. \& McKay, G. Nat. Immunol. 21, 591-594 (2020).

11. Nachega, J. B., Mbala-Kingebeni, P., Otshudiema, J., Zumla, A. \& Tam-Fum, J.-J. M. Lancet Glob. Health 8, e991-e992 (2020).

12. The Global Fund. https://www.theglobalfund.org/en/covid-19/ news/2020-06-17-global-fund-survey-majority-of-hiv-tb-andmalaria-programs-face-disruptions-as-a-result-of-covid-19/ (17 June 2020).

13. Lam, E., McCarthy, A. \& Brennan, M. Hum. Vaccin. Immunother 11, 2627-2636 (2015).

14. Burchett, H. E. D. et al. Vaccine 32, 6505-6512 (2014).

15. Romero, S., Harmon, A., Tompkins, L. \& del Rio, G.M.N The New York Times https://www.nytimes.com/2021/02/04/us/ covid-vaccines-crossing-states.html (4 February 2021).

16. Human Rights Watch. https://www.hrw.org/news/2021/ 01/28/ lebanon-gaps-remain-vaccine-program-planning (28 January 2021).

17. Human Rights Watch. https://www.hrw.org/news/2021/02/02/ syria-covid-19-vaccine-access-should-be-expanded-fair (2021).

18. Lieberman, A. Devex https://www.devex.com/news/

humanitarians-plan-for-covid-19-vaccinations-in-conflictzones-98971 (25 January 2021).

19. Parkin Daniels, J. The Guardian https://www.theguardian.com/ global-development/2020/dec/22/colombia-coronavirusvaccine-migrants-venezuela-ivan-duque (22 December 2020).

20. Welsh, T. Devex https://www.devex.com/news/in-brief-legalstatus-for-venezuelans-in-colombia-to-improve-vaccineaccess-99118 (9 February 2021).

21. Nnadi, C. et al. J. Infect. Dis. 216, S368-S372 (2017).

22. de Quadros, C. Sci. Am. https://www.scientificamerican.com/ article/victories-and-challenges-in-the-war-against-polio/ (2013).

23. Toppenberg-Pejcic, D. et al. Health Commun. 34, 437-455 (2019).

24. Edwards, J.K. et al. MEASURE Evaluation https://www measureevaluation.org/resources/publications/tr-20-412 (2020).

25. Camlin, C.S., Cassels, S. \& Seeley, J. J. Int. AIDS Soc. https://doi. org/10.1002/jia2.25136 (2018).

\section{Acknowledgements}

We thank S. Guyer and K. McCann for research and editorial support. The authors of this Comment are members of a research consortium led by Columbia University's Program on Forced Migration and Health, with the American University of Beirut, Brandeis University, Georgetown University and Universidad de los Andes. The consortium is conducting a two-year research project that aims to provide evidence and guidance to strengthen health systems to address the needs of displaced and host populations in contexts of protracted displacement. The program is funded by UK aid through the Foreign, Commonwealth and Development Office (FCDO). It is managed by the World Bank Group (WBG) and was established in partnership with the United Nations High Commissioner for Refugees (UNHCR). This work does not necessarily reflect the views of UK Aid, the WBG or UNHCR.

\section{Author contributions}

All authors jointly conceived of and conceptualized the commentary; M.Z. led the design and development of the manuscript; M.Z., L.S.L. and M.N.A conducted background research; M.Z. and L.S.L. drafted and revised the manuscript; and all authors reviewed the manuscript and provided several rounds of critical feedback.

\section{Competing interests}

The authors declare no competing interests.

\section{Additional information}

Supplementary information The online version contains supplementary material available at https://doi. org/10.1038/s41591-021-01328-3.

\section{Sustained behavior change is key to preventing and tackling future pandemics}

Investment in research and programs to discover and apply the principles that underpin sustained behavior change is needed to address the continuing threat from COVID-19 and future pandemics and will require collaboration among behavioral, social, biomedical, public-health and clinical scientists.

\section{Susan Michie and Robert West}

1 uman behavior was instrumental in causing COVID-19, and changing it has been vital to tackling this pandemic. The countries that have done best in mitigating COVID-19's harms to health and to their economies have rapidly and successfully persuaded their populations to enact large-scale behavior change. Some of these interventions have been highly effective, others have been less so, and some have produced substantial social and financial harm. In particular, national 'lockdowns' have been effective in keeping people from interacting, to reduce the spread of disease ${ }^{1}$, but they have been very damaging to people's lives and to national economies. Therefore, lockdowns should ideally be used only to bring transmission levels low enough to be controllable by other policies. These policies include adequate 'find, test, trace, isolate and support' systems ${ }^{2}$; border controls and quarantine to prevent reseeding of infections; the creation of safe working, domestic and transport spaces; and the promotion of personal protective behaviors such as the use of face coverings ${ }^{3}$.

Capability, opportunity and motivation Large-scale, sustained behavior change is needed to reduce the risk of, and to prepare for, future pandemics. ${ }^{4}$ The COVID-19 pandemic has shown that populations will adopt at least some of the required behaviors under certain conditions ${ }^{5}$. However, adoption has been variable across countries, over time and across social groups ${ }^{5}$.

Achieving sustained behavior change requires a sound understanding by policymakers and intervention designers of what underpins the behaviors concerned. For example, what does it take in all cultures to ensure that, where appropriate, people keep safe physical distances from each other, wear face coverings masks and disinfect their hands? What is needed to ensure that adequate ventilation is provided in enclosed spaces, and that people in high-risk settings use personal protective equipment effectively?

We provide here important behavioral targets for the prevention and mitigation of 